\section{e0700 THE DIAGNOSTIC VALUE OF DUAL-SOURCE CT IN PATIENTS SUSPECTED OF CORONARY ARTERY DISEASE: A META- ANALYSIS}

doi:10.1136/hrt.2010.208967.700

${ }^{1}$ Li Min, ${ }^{2}$ Zhang Xiaona, ${ }^{1}$ Ma Shaohui, ${ }^{1}$ Zhang Ming. ${ }^{1}$ Imaging Department, First Affiliated Hospital, Medical College of Xi'an Jiaotong University; ${ }^{2}$ Imaging Department, Second Affiliated Hospital, Medical College of Xi'an Jiaotong University

Objective To conduct a meta-analysis-based evaluation of dualsource CT (DSCT) in the diagnosis of coronary artery disease (CAD).

Methods English literatures were searched in Medline. A study was identified if it: (1) used DSCT angiography as a diagnostic test for detection of significant coronary stenosis ( $\geq 50 \%$ diameter stenosis) in patients with suspected CAD, and (2) used coronary angiography as the reference standard. Sensitivity, specificity with $95 \% \mathrm{CI}$ on the patient basis was pooled by bivariate random-effects model. Hierarchical weighted symmetric summary receiver-operating curve was also estimated. Likelihood ratios were calculated on the basis of the pooled estimates of sensitivity and specificity. By means of Bayes' theorem integrated with pooled likelihood ratios, the clinical utility of the results was estimated.

Results 10 studies included 1271 patients were identified. Mean rate of nonevaluable patient and segment was 5.6\% (33/590) and 2.3\% (271/11745) respectively. The pooled results were as follows: sensitivity of $99 \%(97 \%-99 \%)$, specificity of $86 \%(79 \%-90 \%)$, positive likelihood ratio of 6.84 , and negative likelihood ratio of 0.01 . Given a pre-test probability below $84 \%$, the likelihood of disease was less than $5 \%$ based on a negative DSCT. If a pre-test probability was above $13 \%$, the likelihood of disease was more than $50 \%$ on a positive DSCT.

Conclusions DSCT angiography has good diagnostic performance in the detection of $\mathrm{CAD}$, however, it could not replace conventional coronary angiography totally.

\section{e0701 COMPARISON OF FREE-BREATHING 3D AND CONVENTIONAL 2D LATE GADOLINIUM ENHANCEMENT CARDIAC MR FOR THE EVALUATION OF LEFT VENTRICULAR INFARCTION}

doi:10.1136/hrt.2010.208967.701

Ma Ning, Zhao Shihua, Yin Gang, Lu Minjie, Jiang Shiliang, Cheng Huaibing, Liu Qiong, Yang Xinling. Cardiovascular Institute and Fu Wai Hospital

Purpose To compare a new free-breathing 3D late gadolinium enhancement (LGE) cardiovascular magnetic resonance with conventional 2D LGE in patients with prior myocardial infarction. Methods Patients with prior myocardial infarction were studied using a segmented 2D Phase-Sensitive Inversion-Recovery (PSIR) turbo FLASH sequence followed by a navigator-gated 3D PSIR turbo FLASH sequence on a $1.5 \mathrm{~T}$ MR scanner for myocardial late enhancement after the administration of gadopentetate dimeglumine. All images were evaluated by two independent cardiovascular radiologists. Overall image quality was graded. The hyperenhanced myocardial scars were compared quantitatively in volume and scored qualitatively on the basis of size (area) and location.

Results 24 patients were successfully scanned using both approaches. Image quality was considered diagnostic for analysis in 23 cases, and this group underwent qualitative and quantitative scar analyses. $2 \mathrm{D}$ and $3 \mathrm{D}$ results were found to be highly correlated for hyperenhanced scar area and scar location. There was a high correlation between the hyperenhanced lesions volumes determined by $2 \mathrm{D}$ PSIR and 3D PSIR $\left(\mathrm{R}^{2}=0.86\right)$. The average hyperenhancement lesion score (maximum possible score, 68) between both readers based on scar area per patient was 31.85 (range, 8.5-48) for 3D PSIR and 26.91 (range, 8.5-44.5) for 2D PSIR $(\mathrm{p}<0.01)$. The average hyperenhancement lesion score based on scar location (maximum possible score, 34) per patient was 19.46 (range, 3.5-28.5) for 3D PSIR and 16.89 (range, 4.5-26) for 2D PSIR $(p<0.01)$. The mean scar volume over all cases was $31.41 \mathrm{ml}$ (range, 8.6-63.8) for $3 \mathrm{D}$ PSIR and $23.42 \mathrm{ml}$ (range, 7.4-44.6) for 2D PSIR ( $p<0.01)$.

Conclusion Free-breathing 3D PSIR turbo FLASH provides a promising approach for accurate scar quantification and detection of left ventricular myocardial scar particularly for patients who cannot perform repeated breath-holding.

\section{e0702 PREVALENCE OF ANOMALOUS CORONARY ARTERIES}

doi:10.1136/hrt.2010.208967.702

M Lim, L Devaraja, C de Larrazabal. Singapore Medical Specialists Centre, Paragon, Singapore

Objective Coronary arteries with an anomalous origin and course may occasionally cause sudden death. This is the first study of the prevalence of anomalous coronary arteries in an Asian population. Methods Over a 4 year period, consecutive patients who were referred to our centre for coronary CT angiogram were prospectively assessed for the prevalence of anomalous coronary arteries.

Results Over the 4 year period, a total of 4500 were assessed. The prevalence of coronary artery anomalies is $0.78 \%$. Coronary arteries can be considered anomalous as a result of single coronary artery or anomalous origin of coronary arteries. There were six of 4500 who had single coronary arteries of which five had absent right coronary artery (RCA) and one had absent left main coronary artery (LMCA). There were 29 patients who had anomalous origin of coronary arteries: four had anomalous left circumflex artery origin, 20 had anomalous RCA origin, four had origin of LMCA or LAD from RCA and one had origin of LMCA from the right coronary sinus. Hence, the commonest anomaly was anomalous origin of RCA.

Conclusion The incidence of anomalous coronary arteries was $0.78 \%$ and the commonest coronary anomaly was anomalous origin of RCA.

\section{e0703 RADIATION DOSAGE COMPARISON BETWEEN DIFFERENT CORONARY CT ANGIOGRAPHY PROTOCOLS}

doi:10.1136/hrt.2010.208967.703

M Lim, S R Devi, C de Larrazabal. Singapore Medical Specialists Centre, Paragon, Singapore

Objective Prospective ECG gated coronary CT angiography (pECGCCTA) has become an alternative CT protocol. It limits radiation exposure at a narrowed predefined end diastolic time segment instead of during the entire phase of the cardiac cycle, as compared with the retrospective ECG gated coronary CT angiography (rECGCCTA). In this study, we determined the difference in radiation dose between pECG-CCTA and rECG-CCTA

Methods Consecutive patients who underwent CCTA were selected for our study; 29 patients underwent pECG-CCTA and 29 patients underwent rECG-CCTA. Inclusion criteria were Body mass index (BMI) of $\leq 30$, sinus rhythm, $\mathrm{HR} \leq 70 \mathrm{bpm}$. Exclusion criteria were BMI $\geq 30$, irregular rhythm, HR $>70 \mathrm{bpm}$. All the patients were given $\beta$-adrenergic blockers and bromazepam to obtain heart rate of $\leq 70 \mathrm{bpm}$. Patients were given $50 \mathrm{ml}$ of IV Iopamiro 370 at $4.0-5.0 \mathrm{ml} / \mathrm{s}$ and $50 \mathrm{ml}$ saline at $4.0-5.0 \mathrm{ml} / \mathrm{s}$. The tube voltage was constant at $100 \mathrm{Kvp}$ and effective tube current was adapted according to the individual patient's body mass index. Effective radiation dose was calculated for both groups.

Results The mean effective radiation dose for the normal BMI group using pECG-CCTA and rECG-CCTA protocols were $1.7 \pm 0.2 \mathrm{mSv}$ and $2.9 \pm 0.9 \mathrm{mSv}$ respectively. Similarly for overweight BMI group 